\title{
"Spielend" Sprachen lernen? Zur Benutzung der Zweitsprache im Freispiel bei Kindergartenkindern in Frankreich
}

Anemone Geiger-Jaillet et Marita Vagedes Husser

\section{(2) OpenEdition \\ Journals}

Édition électronique

URL : https://journals.openedition.org/esp/917

DOI : 10.4000/esp.917

ISSN : 2532-0319

Éditeur

Centre d'Information sur l'Éducation Bilingue et Plurilingue

Édition imprimée

Date de publication : 1 décembre 2016

Pagination : 13-27

ISSN : 1127-266X

Référence électronique

Anemone Geiger-Jaillet und Marita Vagedes Husser, "Spielend" Sprachen lernen? Zur Benutzung der Zweitsprache im Freispiel bei Kindergartenkindern in Frankreich", Éducation et sociétés plurilingues [Online], 41 | 2016, Online erschienen am: 20 Oktober 2017, besucht am 29 August 2021. URL: http:// journals.openedition.org/esp/917 ; DOI: https://doi.org/10.4000/esp.917 


\section{„SPIELEND“ SPRACHEN LERNEN? ZUR BENUTZUNG DER ZWEITSPRACHE IM FREISPIEL BEI KINDERGARTENKINDERN IN FRANKREICH}

\section{Anemone Geiger-JaIllet, Marita Vagedes Husser}

I ricercatori si sono interessati poco alla misura in cui la scelta delle lingue influisce sull'identità e sullo status sociale di un bambino in età prescolare. Le conversazioni fra bambini ci sembrano spesso banali, ma per il bambino questi scambi sono molto importanti. I bambini creano i loro propri rituali, le regole e le condizioni di partecipazione ad un gioco. In quest'articolo si tratta della scelta della lingua da parte del bambino in funzione delle sue intenzioni di comunicazione, degli interlocutori e dei loro livelli di lingua rispettivi. Si tratta di bambini fra $i 5$ e $i 6$ anni frequentanti una struttura di scuola materna a parità oraria all'interno della rete associativa A.B.C.M. Zweisprachigkeit in Alsazia.

Schlüsselbegriffe: gioco libero (accompagnato), insegnamento precoce, cambiamento dinamico della lingua, identità bilingue, identità sociale

Researchers have not paid much attention to the ways language choice may affect the identity and social status of pre-schoolers. Conversations between children often seem banal to us, but for a child such exchanges are very important. Children create their own rituals and rules to have the right to enter a game. This article deals with the children's choice of language depending on what they intend to communicate, on their interlocutors and on their respective language levels. Of 5 to 6 years of age, they are attending a kindergarden run by the association A.B.C.M. Zweisprachigkeit in Alsace, where both French and German are taught on a par.

key-words: child-initiated play, early teaching, dynamic code-switching, bilingual identity, social identity

EINLEITUNG UND KONTEXT DER BEOBACHTUNGEN
Tnstitutionelle frühe Mehrsprachigkeit benutzt in Anlehnung an familiäre Zweisprachigkeit oft den pädagogischen Ansatz der Immersion, das „Eintauchen“ in die Sprache mit muttersprachlichen Erwachsenen. Diese nehmen gegenüber den Kindern/Schülern die Rolle des zielsprachlichen - aber monolingualen - Sprechers ein.

In den folgenden Beispielen aus dem privaten bilingualen Kindergarten Les Mickele in Frankreich wird das Modell „Ein (muttersprachlicher) Lehrer - eine Sprache" mit Deutsch und Französisch praktiziert. Dennoch trauen sich die Kinder im Rahmen des begleiteten Freispiels, in Anwesenheit der Lehrerin 
„Spielend“ Sprachen lernen? A. Geiger-Jaillet,
M. Vagedes Husser (die das Deutsche repräsentiert), auch Französisch zu reden.

Die individuellen Sprach(en)biographien der Kinder ergeben, dass etwa 30\% frankophone einsprachige Schüler 2015-16 diese Schule besuchten, alle anderen hatten mindestens zwei Sprachen im häuslichen Umfeld. Französisch ist die Umgebungssprache in Strasbourg und in der Einrichtung Les Mickele - eine Sprache, die als lingua franca eingesetzt werden kann, um von allen verstanden zu werden. Innerhalb der beobachteten Kleingruppen von maximal drei Kindern erfassen wir Momente, in denen dynamische Sprachwechsel zwischen Deutsch und Französisch stattfinden, und fragen uns: Wodurch lassen sich die Kinder in ihrer Sprachwahl beeinflussen? Ist der für das Kind jeweils motivierte Sprachwechsel für Erwachsene nachvollziehbar? Gibt es Auslöser oder Regelmäßigkeiten? Wird die Zielsprache Deutsch, die für die meisten Kinder die Zweitsprache ist, auch bei ihren Spielaktivitäten unter peers benutzt?

Die Daten zur Untersuchung wurden von Vagedes (2014) im Rahmen einer Masterarbeit aufgezeichnet. Die Straßburger Mickele-Schule ist eine Vorschule in Trägerschaft des Elternvereins A.B.C.M. Zweisprachigkeit, die Kinder von zweieinhalb bis 6 Jahren betreut. Sie ist deutsch-französisch ausgerichtet, nicht nur vom Kontakt der Sprachen her, sondern auch, was das Lehrpersonal, die Schülerschaft und den pädagogischen Ansatz betrifft, welcher Elemente der französischen VorSCHULE mit denen eines deutschen KinderGARTENS vereint (cf. Geiger-Jaillet 2010b). Im Sinne der reziproken Immersion gilt es, die frühe Zweisprachigkeit und die Bikulturalität $\mathrm{zu}$ fördern. In Kleingruppen von 2-3 Kindern wurden neun Spielsituationen mit Video aufgenommen (Gesamtdauer 101 Minuten) und mit der Software Exmaralda transkribiert. Diese beruhten überwiegend auf Konstruktions- oder Rollenspielen mit Lego-Bausteinen oder Playmobil-Spielfiguren. Ergänzend wurde teilnehmende Beobachtung mit anschließendem Protokoll durchgeführt, und die Daten eines ausführlichen Anmeldebogens mit Beschreibung des Sprachprofils der jeweiligen Familie wurden zur Interpretation einbezogen.

In dieser Schule gelten gewisse Spielregeln, auf die sich alle einlassen: die Lehrperson spricht gegenüber den Kindern nur die Zielsprache Deutsch, und auch, wenn sie die französischen Äußerungen der Kinder wohl versteht, stellt sie sich (im Sinne des oben zitierten immersiven Ansatzes) gegenüber den Kindern als Bezugsperson für die deutsche Sprache dar. Diese „künstliche Situation" wird aber von allen Beteiligten akzeptiert, und moti- 
„Spielend“ Sprachen lernen? A. Geiger-Jaillet,
M. VAgedes Husser

SPRACHENVERMITT LUNG UND SPIEL IN DER VORSCHULE viert die Kinder, sich in der Zielsprache auszudrücken.

Das Beispiel der Mickele-Schule zeigt, dass der methodische Ansatz des immersiven Sprachbads nicht mit der Kontaktzeit eines Kindes mit der Zielsprache zu verwechseln ist. Da eine Lehrperson in einer Gruppe mit 25 Kindern nicht überall gleichzeitig sprachlichen Input auf Deutsch geben kann, bilden sich sowohl bei gelenkten als auch bei spontanen Aktivitäten Parallelgruppen mit Kind-zu-Kind-Kommunikationen. Dabei sind die zwei Sprachen Deutsch und Französisch häufig gleichzeitig im Gruppenraum zu hören - obwohl gerade offizielle ,deutsche Zeit" ist. Angesichts dieser Situation sollte für die frankophonen Schüler die Kontaktzeit mit ihrer "schwacher Sprache“ Deutsch nach Möglichkeit erhöht werden. Informelle Zeiten wie das gemeinsame Mittagessen (Kantine) und der Pausenhof sind den Kindern in dieser Hinsicht sehr wichtig. Gerade den Kindern, die Deutsch als Zweitsprache lernen, bringt die Erfahrung viel, wenn sie sehen, dass andere Kinder - und nicht nur erwachsene Betreuungs- bzw. Lehrpersonen - spontan beim Spielen und in sonstigen Interaktionen die deutsche Sprache verwenden.

Die Eltern suchen nach einer anregenden pädagogischen Umgebung, die optimale Entwicklung des eigenen Kindes steht im Vordergrund (vgl. Dompmartin-Normand 2002: 42.). Mit der Wahl einer bilingualen Einrichtung verbinden die Eltern oftmals die Vorstellung, dass ihr Kind ,spielend“ lernt, eine prestigeträchtige Zweitsprache fließend zu sprechen.

Dennoch erfolgt der frühe Erwerb einer neuen Sprache nicht so einfach und natürlich wie der Erwerb einer Muttersprache. Die privilegierten Bedingungen des sprachlichen „AufeinanderEingehens" in der Familie (joint attention) lassen sich in einer Institution nicht nachahmen, wenn sich das Kind in einer Gruppe von 25 -30 Kindern befindet.

In Europa sind Vorschulprogramme mit dem Ziel, eine Zweitsprache wie z.B. Englisch, Französisch oder Deutsch durch den täglichen Kontakt mit einer muttersprachlichen Erzieherin zu erwerben, schon recht verbreitet. I In Frankreich gibt es ferner Intitiativen, durch immersiven Unterricht den Erhalt einer Regionalsprache zu fördern oder die Zweisprachigkeit einer Grenzregion aufzuwerten (cf. Geiger-Jaillet 2010a).

Dabei sind die Erwartungen und Ansprüche an solche Programme seitens der Sprachenpolitiker, Institutionen, Pädagogen, Eltern und Kinder nicht immer deckungsgleich ... 
„Spielend“ Sprachen lernen? A. Geiger-Jaillet,
M. Vagedes Husser
Politisch Verantwortliche erwarten z.B. von einem Schulversuch oder einem bilingualen Schulmodell, dass die Regionalsprache auf jeden Fall in der heranwachsenden Generation erhalten werden soll.

Um die Kinder dazu zu bringen, eine Regionalsprache wie z.B. Katalanisch zu benutzen, soll in den Augen manch Verantwortlicher die dominante Umgebungssprache während des Schulalltags möglichst, ausgeschaltet" werden. So wird im Konzept der privaten Bressola-Schulen (Internetauftritt von Bressola http://www.bressola.cat/fr_labressola) ausdrücklich verlangt, dass der katalanisch sprechende Lehrer die Kinder am Anfang nicht zu Wort kommen lässt, damit sie nicht ins Französische ,verfallen“.

Mais ce qui n'est pas si évident c'est d'installer l'habitude de la communication spontanée en catalan entre les enfants qui sont dans cette situation. Evidemment ils s'exprimeront d'abord en français. Aussi devons-nous être attentifs à bien couvrir le maximum d'espaces sonores disponibles, au moins pendant les premières semaines de l'année scolaire... Il est très important, pendant les premiers mois de l'année scolaire, de limiter au maximum les coins libres et que l'enseignant adopte un rôle très protagoniste dans la classe. (Franquesa 2013/2015, ohne Seitenangabe)

Im Gegensatz zur katalanischen Situation, die Franquesa (2013/2015) beschreibt, hätte das Eingreifen mit dem Ziel, eine bei den Schülern präsente bzw. dominante Sprache zu unterdrücken (!), unserer Meinung nach im deutsch-französischen Grenzraum keine oder nur eine sehr begrenzte Wirkung.

Die Erwartungen der Institution (in diesem Falle der Schulbehörden) und der Eltern sind häufig auf eine frühzeitige Sprachproduktion gerichtet. Rezeptive Kompetenzen und metasprachliches Bewusstsein sind weniger gut greifbar und daher In der Unterrichtsforschung viel weniger beschrieben. Aber Sprachvergleich, metasprachliche Kompetenzen und die Ausbildung einer doppelten Weltsicht (1) verändern den Menschen.

Ein starres Beharren auf dem Prinzip „Eine Person - eine Sprache" wird heute, 100 Jahre nach Jules Ronjats Ansatz, von vielen Pädagogen als nicht mehr als zeitgemäß angesehen.

Im folgenden Beispiel sieht man deutlich, wie das sprachliche Eingreifen der Lehrperson, die auf einer Erklärung in deutscher Sprache besteht, dazu führt, dass die Spielsequenz abbricht. 
BEISPIEL 1

(TR1301.68-70) NICHTVERSTEHEN (DAMIEN 5;9)

\begin{tabular}{|l|l|}
\hline \multicolumn{2}{|l|}{$\begin{array}{l}\text { Damien und } 2 \text { andere Kinder spielen mit selbstgebauten Legofahrzeugen. Damien hat einen kleinen Arztkoffer } \\
\text { gefunden. Er wendet sich an die Lehrperson, um ihr etwas zu zeigen. }\end{array}$} \\
\hline Damien & Il faut ça dans le-, un coffre, en cas où il se fait mal. \\
\hline Lehrperson & Bitte? \\
\hline Damien & Faut ça dans le coffre, en cas où il se fait mal. \\
\hline Lehrperson & Was hast du in den Kofferraum gemacht bei deinem Traktor? \\
\hline Damien & $(--)$ \\
\hline
\end{tabular}

„Spielend“ Sprachen lernen?
A. Geiger-Jaillet,
M. VAGEDES HUSSER

Der Pädagoge macht hier die Erfahrung, dass sich Sprache im Spiel nicht verordnen lässt, sondern er/sie Spiel nur begleiten kann. Das Kind behält die Möglichkeit, keine Antwort zu geben oder die Kommunikation bzw. Interaktion ganz abzubrechen. Wie kann eine Lehrkraft nun damit umgehen, dass im Klassenraum immer mehrere Sprachen präsent sind, selbst wenn sie nur in einer unterrichtet?

Pädagogen, die die Kinder und ihre Bedürfnisse ernstnehmen, werden versuchen, der Perspektive der Kinder vorzugreifen: für welche Handlungen im Kindergarten ist die Zweitsprache sinnvoll und welche sollten vielleicht der Erstsprache vorbehalten bleiben? Dabei sind gerade auch die von den Kindern selbst organisierten Interaktionen ein zentraler Punkt, stellt sich doch die Frage, ob und wie Spracherfahrungen mit peers beim Zweitspracherwerb genutzt werden können.

Bisher war es in Frankreich eher unüblich, dass die Vorschullehrkraft die Kinder in Freispielsituationen begleitet oder sogar aktiv mitspielt (vgl. Geiger-Jaillet 2010: 127). In anderen Ländern wie Deutschland, der Schweiz oder Kanada wird der Spielbegleitung im Kindergarten ein hoher Stellenwert für kindliche Lernprozesse eingeräumt (play tutoring).

In Frankreich findet in dieser Hinsicht nun ein Paradigmenwechsel statt. Im Lehrprogramm des französischen Ministeriums von 2015 finden sich einige sehr neue Töne, wie der folgene Zwischentitel «Apprendre en jouant» (übersetzt „Durch Spielen lernen") bekundet:

Le jeu favorise la richesse des expériences vécues par les enfants dans l'ensemble des classes de l'école maternelle et alimente tous les domaines d'apprentissages. Il permet aux enfants d'exercer leur autonomie, d'agir sur le réel, de construire des fictions et de développer leur imaginaire, d'exercer des conduites motrices, d'expérimenter des règles et des rôles sociaux variés. Il favorise la communication avec les autres et la construction de liens forts d'amitié. Il revêt diverses formes: jeux symboliques, jeux d'exploration, jeux de construction et de manipulation, jeux collectifs et jeux de société, jeux fabriqués 
„Spielend“ Sprachen lernen? A. Geiger-Jaillet,
M. Vagedes Husser et inventés, etc. L'enseignant donne à tous les enfants un temps suffisant pour déployer leur activité de jeu. Il les observe dans leur jeu libre afin de mieux les connaître. Il propose aussi des jeux structurés visant explicitement des apprentissages spécifiques. (MEN 2015, B.0. 26.03. 2015: Teil «2.1. Apprendre en jouant»)

Beim Spiel in der Kindergruppe lernt das Kind nicht nur eigenständig zu planen und zu handeln, es entwickelt auch sprachliche und soziale Strategien, die es ihm ermöglichen, die eigenen Bedürfnisse und Vorstellungen mit anderen auszutauschen. Sprache in Verknüpfung mit Spiel führt für das Kind zur ersten Ausprägung einer sozialen Identität.

Aus der Sicht der Kinder ist es also von Bedeutung, welche Sprache gewählt wird. Zugleich bietet der Rahmen einer Kind-zu-KindInteraktion viel mehr sprachliche Lernmöglichkeiten und eigenständiges Handeln als einfache Lehrer-Schüler-Dialoge, die oft nur aus Anweisungen in Imperativ-Form oder einer einfachen Frage-Antwort-Sequenz bestehen.

Durch Peer-Interaktion lernen Kinder vieles: Sie entwickeln die Fähigkeit, Dinge aus der Perspektive einer anderen Person zu sehen. Sie lernen, Kompromisse zu machen, um Konflikte zu lösen [, sie lernen] zu teilen und mit anderen zusammenzuarbeiten. Kinder lernen so auch zu verhandeln und sich zu behaupten, ebenfalls wichtige Fähigkeiten. All dies wird durch die Verwendung von Sprache erreicht. Üblicherweise finden diese Peer-Interaktionen im Rahmen von Spielsituationen statt. (Licandro/Lüdtke 2013: 3)

Besonders altersgemäße Spielformen sind Konstruktions- und Rollenspiele. Konstruktionsspiele trainieren die feinmotorischen Fähigkeiten, z.B. aus Legosteinen ein Haus zu bauen, denn „Konstruktionsspiel ist immer mit gegenständlichem Handeln verbunden“. Hierfür ist ,kommunikative Verständigung zur Koordination der konstruktiven Tätigkeit erforderlich" (Andresen 2002: 178), wie wir später sehen werden.

Das Rollenspiel als interaktive Spielform unter mehreren Kindern ist besonders im Alter zwischen 4 und 7 Jahren beliebt. Rollenspiele sind sprachlich anspruchsvoller und für Lerner mit geringen zielsprachlichen Kenntnissen schwerer zu realisieren. Für den Spielablauf ist es von großer Bedeutung, dass die Kinder sich mit ihren Spielpartnern verständigen können. Bei komplexeren Formen des Rollenspiels ist die sogenannte Metakommunikation nötig (siehe auch Andresen 2002: 175).

Im nächsten Abschnitt werden wir versuchen, diese kommunikative Verständigung und das nötige Sich-Verständigen-Können im Spiel in den Zusammenhang mit dem Erlernen einer Zweitsprache zu stellen. 
„Spielend“ Sprachen lernen? A. Geiger-Jaillet,
M. Vagedes Husser

MITSPIELEN: SPRACHLICHER AUSTAUSCH UNTER KINDERN EINER BILINGUALEN KINDERGRUPPE

\section{DIE KINDER} KÖNNEN DIE ZIELSPRACHE BENUTZEN
Wenn im Folgenden einige Beispiele von geleitetem und freien Spielsequenzen mit Lego und Playmobil aus einem bilingualen deutsch-französischen Kindergarten in Strasbourg aufgeführt werden (cf. Vagedes 2014), so ist zu berücksichtigen, dass alle Kinder, wie schon oben angedeutet, eine sehr individuelle Sprach(en)biographie haben: Durch den Besuch des Kindergartens verfügen alle über eine Kontaktzeit von mindestens zwei Jahren mit der Sprache Deutsch, einige Kinder sprechen auch Deutsch mit Ihren Eltern zuhause oder wohnen grenznah in Deutschland, manche haben bereits eine zweisprachige Krippe besucht.

Interessant sind nun die Berührungspunkte, die im Spiel zwischen Kindern mit verschiedenen Sprachbiographien entstehen, die Wechsel von einer Sprache zur anderen, und natürlich die Frage, wie sich die Kinder auf die Verwendung der einen oder anderen Sprache einigen.

Wir werden nun einige Kategorien untersuchen, bei denen sich Gesetzmäßigkeiten für einen Sprachwechsel erkennen lassen. Zunächst führen wir Beispiele auf, in denen die 5-6jährigen Kinder die Zweitsprache/Zielsprache Deutsch beim Spiel im Vorschulalltag benutzen.

Durch die Bindung an eine „einsprachige“ Bezugsperson kann das Kind eine emotionale Bindung zu der Sprache aufbauen. Häufig ist es so, dass das Kind dann die neue Sprache der Bezugsperson zuliebe benutzt, was in unserer Untersuchung (Vagedes 2014) bestätigt wurde: Die Kinder wählten zu 96\% deutsche Formulierungen, wenn sie sich im Spiel an die deutsche Lehrperson wandten.

Es ist bekannt, dass Kinder beim Erwerb einer Zweitsprache zuerst kurze formelhafte Sätze benutzen, also Formeln der

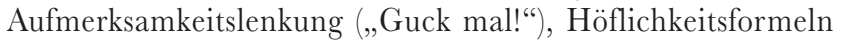
(„Danke!") und Gesprächsroutinen wie ,Ja/Nein“ (vgl. Wörle 2013). Solche chunks können deutschlernende Kinder durchaus in das Spiel integrieren, wenn sie zum Beispiel ihre Rollenspielfigur „Hallo, hallo!“ rufen lassen. Manchmal wird mit Pseudowörtern versucht, den Klang einer deutschen Sprachmelodie nachzuahmen.

Ist die Lehrkraft nicht anwesend, haben wir Belege für den ausschließlichen Gebrauch des Französischen der beiden Mächen Isabelle (die zuhause zweisprachig aufwächst) und Nadia (aus einer frankophonen Familie). Anscheinend reicht schon die Präsenz der deutschsprachigen Lehrperson in Beispiel 2 aus, 
„Spielend“ Sprachen lernen?

\section{A. GEIGER-JAiLlet, \\ M. VAGEDES HUSSER}

damit Isabelle sich nun auf Deutsch als Kommunikationssprache einstellt. Nadia passt sich an, kann aber nur kleinere Beiträge im Sinne von chunks einbringen und keine so umfangreichen Kommentare wie ihre Kameradin. An der Stelle, an der sie etwas sagen möchte, aber nicht die Worte findet, wird dies sehr deutlich.

\section{BEISPIEL 2 (TR0801) CHUNKS EINBRINGEN \\ ISABELLE (5;8) UND NADIA (5;4)}

\begin{tabular}{|c|c|}
\hline Isabelle & Und dein Mann! (Isabelle gibt Nadia die Legofigur) \\
\hline Isabelle & (zur Lehrperson) Du fotografierst, wenn wir rutschen. \\
\hline Nadia & Ja. \\
\hline Isabelle & Eins-zwei-drei! \\
\hline Nadia & .... drei! (Nadia spricht im Chor mit Isabelle) \\
\hline Lehrperson & Jetzt sind sie runter gerutscht, \\
\hline Nadia & Und guck mal! Der hat da... \\
\hline Isabelle & $\begin{array}{l}\text { Jetzt geh ich mal nach Hause, auf*mein Pferd. Und geh mal auf mein Pferd, das } \\
\text { Wasser trinkt. (Isabelle setzt ihre Figur auf das Pferd) }\end{array}$ \\
\hline Nadia & Ja, das ist sehr schön. \\
\hline Isabelle & Oh, ich will auf *mein Baum mal klettern. \\
\hline Nadia & (beobachtet die Aktion von Isabelles Figur, öffnet den Mund, sagt aber nichts) \\
\hline Isabelle & Ups! Oh nein! (der Baum bricht auseinander) \\
\hline
\end{tabular}

Aber auch komplexerer Wortschatz kann im Rollenspiel wieder eingesetzt werden, wie Beispiel 3 zeigt. Hugo übernimmt den Begriff „Lanze“, denn er kennt dieses Wort aus dem Unterricht, wo zur Zeit gerade das Thema „Leben bei den Rittern“ behandelt wird.

\section{BEISPIEL 3}

\section{(TR2606.10-15): WORTSCHATZ AUS DEM UNTERRICHT EINBRINGEN (YANNICK 5;11) \\ UND HUGO $(6 ; 0)$}

\begin{tabular}{|l|l|}
\hline \multicolumn{2}{|l|}{ Spiel mit der Ritterburg, Hugo spielt mit einer kleinen Lanze. Yannick entdeckt auch eine Waffe. } \\
\hline Yannick & He, j'ai la même, j'ai la même! \\
\hline Hugo & Que celle-là? \\
\hline Yannick & Oui. xx la mort xx. \\
\hline Hugo & $\begin{array}{l}\text { Regarde: Eine Lanze . Bumm. Chch. Regarde: Eine Lanze } \\
\text { [beim zweiten Mal gesungen]. Bumm. Chch. } \\
\text { (Hugo zeigt noch zweimal die Geste, wie er sich die Lanze in den Bauch stößt und spricht } \\
\text { bzw. singt dazu einen Teil aus dem Lied "Ritter Klipp") }\end{array}$ \\
\hline
\end{tabular}


„Spielend“ Sprachen lernen?

A. Geiger-Jaillet,

M. VAGEDES HuSSER
Eine besondere Strategie, die Imitationsstrategie, besteht darin, ganze Floskeln des deutschsprachigen Spielpartners nachzuahmen:

BEISPIEL 4 (TR2805.287-292) IMITATIONSSTRATEGIE (HUGO 5;11) UND FELIX (6;3)

\begin{tabular}{|l|l|}
\hline Lehrperson & Die zwei Rennfahrer streiten sich? \\
\hline Felix & $\begin{array}{l}\text { Aber, aber meiner, der ist einer. Der, der, der, der, der, der wird, der wird sein Auto klauen, } \\
\text { aber der schafft es nicht. Der springt ganz schnell in sein Rennfahr- und saust los. } \\
\text { (Felix erklärt eine Rollenspiel-Idee. Er beginnt, zu stottern) }\end{array}$ \\
\hline Hugo & Ja. \\
\hline Lehrperson & Okay. \\
\hline Felix & Hey Mann, lass mich in Ruhe [mit Rollenspielstimme]. \\
\hline Hugo & Hey Mann, lass mich in Ruhe [mit Rollenspielstimme]. \\
\hline Felix & Attends! \\
\hline Felix & Eh, wo hast du das genommen? \\
\hline
\end{tabular}

Hugo, der in einer rein französischsprachigen Familie lebt, kann zuerst nur ein kurzes ,Ja!" beisteuern, um der von Felix kommentierten Situation zuzustimmen. Später nimmt Hugo einen Satz von Felix als Ganzes wieder auf und übernimmt auch dessen Tonfall. Beide bewegen ihre Legofigur im Rollenspiel dazu. Die beiden Lego-Männchen stehen sich gegenüber. Hugo kann somit einen vollständigen passenden Satz in der Zielsprache beitragen und sich perfekt in die Situation „Rollenspiel auf Deutsch“ einfügen.

Gemischtsprachliche Beiträge lassen sich in der gesamten Untersuchung (Vagedes 2014) in der Interaktion mit der Lehrerin $(2 \%)$ und mit anderen Kindern $(4 \%)$ nur selten nachweisen. Die Kinder wechseln im Verlauf einer Spielsequenz zwar die Sprache zwischen verschiedenen Beiträgen (turns), beachten dabei aber durchaus Regeln: Die Sprache im Spiel ändert sich dann, wenn auf eine neue Situation oder einen neuen Spielpartner Bezug genommen wird.

RÜCKGRIFF AUF DIE STARKE SPRACHE L1 FRANZÖSISCH
Im folgenden Abschnitt gehen wir der Frage nach, warum die Kinder manchmal nicht die erwünschte Zielsprache Deutsch beim Spielen benutzen.

Wie schon erwähnt, gebrauchen die Kinder ihre L1 für ihre alltäglichen kommunikativen Bedürfnisse, denn Sprache ist die Schlüsselkompetenz, um am sozialen Leben der Gruppe teilzuhaben. Für die Kinder ist der Kindergarten ein Lebensraum und nicht nur ein Ort, an dem sie Unterrichtszeit verbringen. 
„Spielend“ Sprachen lernen? A. Geiger-Jaillet,
M. Vagedes Husser
Bei Dompmartin-Normand (2002: 40) erwähnen die Schüler mehrmals in den Interviews, dass sie die Regionalsprache Okzitanisch zwar im Unterricht mit der Lehrperson verwenden, nicht aber bei Spielen im Pausenhof oder unter Gleichaltrigen. Das können wir mit unseren Beobachtungen bestätigen. Beim Spielen in Kleingruppen (sei es auf dem Pausenhof oder im Klassenraum) - und ohne Präsenz einer erwachsenen SprachBezugsperson - schwenkt die Sprache meist zur besser beherrschten L1.

Ein wesentlicher Unterschied zwischen Erwachsenen-KindDialogen und Gesprächen unter Gleichaltrigen ist das Tempo. Erwachsene können das Kind mit spezifischen Korrektur- und Modelliertechniken unterstützen, indem sie zum Beispiel die kindlichen Intentionen versprachlichen. Andere Kinder als Gesprächspartner werden eher selten so einfühlsam und abwartend vorgehen. Bei der Kommunikation mit Gleichaltrigen muss das Kind dem Spielpartner seine Intentionen verbal und nonverbal „in Echtzeit" vermitteln können.

Damit kommen wir zum Begriff der Effizienz. Das Kind wechselt in die Sprache, in der es seine Absichten am besten durchsetzen kann, wenn es zum Beispiel darum geht, Spielanteile auszuhandeln, oder wenn die Rollen der Spielteilnehmer verteilt werden. Gerade im Rollenspiel muss die verwendete Sprache besonders explizit sein, um Bedeutungszuweisungen und fiktive Zusammenhänge zu erschaffen und den Spielverlauf im Voraus zu organisieren. Das Kind benötigt die Fähigkeit, eine kontextunabhängige Sprache zu benutzen, um bei diesem Austausch von Ideen mithalten zu können. Mit einer solchen Metakommunikation wird eine zweite Ebene geschaffen, die parallel zu der eigentlichen verbalen oder nonverbalen Spielhandlung steht. Dabei sind die Verhandlungen, wer was übernimmt, schon Teil des Spiels und nehmen fast mehr Zeit ein als das spätere Ausagieren (vgl. Andresen 2002: 108).

Im folgenden Rollenspiel erkennt man deutlich die beiden Ebenen. Auf der Ebene des realen Spiels wird eine Spielsprache benutzt, die Figuren werden mit lautmalerischer Unterstützung bewegt. Zur zweiten Ebene, der Meta-Ebene, gehören Gesprächsabläufe, bei denen über das Spiel diskutiert wird. Die nächsten Handlungsschritte werden vorausgeplant und abgesprochen. 


\section{BEISPIEL 6 (TR1705.256) CODESWITCHING, UM DEN SPIELVERLAUF ZU BEEINFLUSSEN (TIMO 5;8) UND RAPHAEL (6;1)}

\begin{tabular}{|l|l|}
\hline \multicolumn{2}{|l|}{$\begin{array}{l}\text { Rollenspiel mit Legofiguren. Der Prinz kommt, um die Prinzessin zu rauben. Bei der Prinzessin hält sich } \\
\text { noch ein Cowboy auf, um sie zu beschützen. }\end{array}$} \\
\hline Lehrperson & Ah, jetzt kämpfen sie? \\
\hline Raphael & Hüah! \\
\hline Raphael & $\begin{array}{l}\text { Wo ist der jetzt hin? [Atemgeräusche, nachgemacht] } \\
\text { (Der "Prinz" ist vom Pferd gestiegen und rennt den Berg hoch) }\end{array}$ \\
\hline Timo & $\begin{array}{l}\text { Phh! [Geräusch eines Zusammenstoßes beim Kampf] } \\
\text { (Timo schaut Raphael beim Rollenspiel zu und provoziert dann einen } \\
\text { Zusammenstoß mit seiner Legofigur "Prinzessin") }\end{array}$ \\
\hline Raphael & Oui mais elle, elle ne pouvait pas le tuer. \\
\hline Timo & Pourquoi? \\
\hline Raphael & $\begin{array}{l}\text { Parce que. Et maintenant c'était moi. Maintenant tu dois me la donner parce que je te } \\
\text { l'avais dit. } \\
\text { (Raphael kommt mit seiner Figur "Prinz", um die Figur "Prinzessin" zu nehmen) }\end{array}$ \\
\hline Raphael & Sinon, je te mettais en prison. \\
\hline Timo & Et tu pourrais jamais! On disait. \\
\hline Raphael & Oui, mais si j'avais une Falle ici. \\
\hline & $\begin{array}{l}\text { Also-. } \\
\text { (Die Lehrkraft versucht erfolglos, in die Planung des Rollenspiels } \\
\text { einzugreifen). }\end{array}$ \\
\hline Raphael & Alors tu me la donnes ou je te mets en prison. \\
\hline Timo & Jamais. \\
\hline Lehrperson & Ich-, \\
\hline Raphael & $\begin{array}{l}\text { Mais Timo! Aber die war doch im Gefängnis! Und die bleibt } \\
\text { im Gefängnis }\end{array}$ \\
\hline Lehrperson & Darf sie jetzt nicht raus, wenn der Prinz gekommen ist? \\
\hline Timo & (Timo nimmt schnell das Lego-Pferd weg) \\
\hline
\end{tabular}

„Spielend“ Sprachen lernen? A. Geiger-JAillet,
Für die Meta-Ebene wird nach einem Codeswitching die französische Sprache benutzt. Der Gebrauch des Präteritums sowie Formeln wie ",je te mettais en prison/ on disait/ elle ne pouvait pas le tuer" sind in der französischen Sprache als sogenanntes ,conditionnel des règles du jeu" charakteristisch für diese Ebene im Rollenspiel. Gute Sprachkompetenzen sind für die Bildung von Nebensätzen erforderlich. Für eine äquivalente Konstruktion in der Zielsprache Deutsch müssten die Kinder den Konjunktiv anwenden können. Raphael sichert sich einen Spielvorteil, indem er später wieder nach Deutsch zurückwechselt. Da Timo noch nicht so gut Deutsch kann, hat R. ihn damit von der Diskussion über den Spielverlauf ausgeschlossen. Timo weiß sich jedoch mit einer nonverbalen Strategie zu helfen und sichert sich eine wichtige Spielfigur, indem er sich das Pferd nimmt.

Es zeigt sich, dass das Rollenspiel der Kinder eine komplexe 
„Spielend“ Sprachen lernen?

\section{A. Geiger-Jaillet, \\ M. VAGEDES HUSSER}

soziale Situation ist. Sie wählen, ob sie ihre Sprache dem Mitspieler anpassen, oder eben nicht. Die Situation muss jedes Mal neu ausgelotet werden. In einer bilingualen Kindergruppen können Rollenspiele auch in einem bilingualen Modus durchgeführt werden. Verschiedene Sprachen können dabei verschiedenen Figuren zugeteilt werden oder eine bestimmte Ebene im Spiel charakterisieren. Die Sprachwechsel sind in diesem Fall Ausdruck einer bilingualen Identität.

\section{BEISPIEL 7 (TR2106.204-223) SPIELEN IM BILINGUALEN MODUS \\ (ISABELLE $(6 ; 1)$; \\ EMMA (6;4), ISABELLE (6;1) UND DAMIEN (6;3)}

\begin{tabular}{|c|l|}
\hline Isabelle & Wir sagten, dass du eine Verletzung hättest. \\
\hline Emma & Aua! [mit wimmernder Stimme] \\
\hline Isabelle & $\begin{array}{l}\text { Meine Liebste! Ich trag dich in dein Bett! [mit Rollenspielstimme]. Und dann komm } \\
\text { ich. }\end{array}$ \\
\hline Emma & Und jetzt waren die alle beide - Au, Aua! Oh Manno! \\
\hline Isabelle & Oh Manno! \\
\hline Emma & Du bist schwer. \\
\hline Isabelle & Au, au, au. \\
\hline Damien & Baff! [Geräusch eines Zusammenstoßes]... \\
\hline Isabelle & $\begin{array}{l}\text { Guck nur, jetzt sind alle runtergefallen. } \\
\text { Dann haben sie gesagt: Manno, du hast das gemacht, } \\
\text { du hast das gemacht! }\end{array}$ \\
\hline Emma & Nein, du, du du, du du. \\
\hline Isabelle & Du, du du! [lauter Streit der Figuren] \\
\hline Emma & Eh, regardez, on fait ça: [Aktion mit der Figur]. \\
\hline Isabelle & Regarde! xx fait ça Mlmlmlml [wildes Schüttelgeräusch]. \\
\hline Emma & Non, regardez! Mettez vos mains- \\
\hline Isabelle & Alles klar. \\
\hline Emma & Elle descend, elle descend! \\
\hline
\end{tabular}

SCHLUSSGEDANKEN „ÜBER SPRACHE REDEN"
Gemeinsames Spielen bildet einen Rahmen, in dem peers und Erwachsene das Kind beim Erwerb der Zweitsprache unterstützen können. Mit Hilfe von (Spiel)Material findet eine Verständnissicherung über den Kontext statt; durch das Erschaffen fiktiver Zusammenhänge wird das Kind zu kontextgelösten Gesprächen herausgefordert.

Für den funktionalen Gebrauch mehrsprachiger Ressourcen ist es entscheidend, dass die Kinder die Sprachen einsetzen können, um ihre Redeabsicht zu verwirklichen. Entscheidend ist also, ob sich ein Kind über die deutsche Sprache in einen sozialen Kontext wie gemeinsames Spielen befriedigend einbringen kann. 
„Spielend“ Sprachen lernen? A. Geiger-Jaillet,
M. VAgedes Husser
Dabei ist die grammatische Korrektheit oder die Länge der Äußerungen zweitrangig. Selbst wenn die Äußerungen in deutscher Sprache kürzer, einfacher strukturiert oder sogar fehlerhaft sein sollten, erfüllen sie doch ihren Zweck, solange damit in der mehrsprachigen Gruppe eine kommunikative Situation bewältigt werden kann (vgl. Wörle 2013: 103-104). Auch dieses Bewusstsein schafft einigende und kontrastive Verbindungen zwischen sprachlichen Formen, die den Lernenden letztlich als Hilfe dienen (vgl. Forlot 2006).

Wenn außerdem Rückgriffe auf die Erstsprache nicht als Fehler, sondern als Kommunikationsstrategie angesehen werden, eröffnet sich eine neue Perspektive für den immersiven Sprachunterricht. Die Lehrperson kann signalisieren, dass dynamische Sprachwechsel im Sinne von Ofelia Garcías translanguaging als Teil einer wachsenden bilingualen Identität der Kinder willkommen sind. Damit eröffnen sie neue metalinguistische Lernmöglichkeiten. So wird nicht nur der Gebrauch der deutschen Sprache gefördert, sondern das allgemeine Sprachwissen der Kinder.

Eine bewusste Sprachbetrachtung ist mit dem Heraustreten aus der Spielsituation verbunden. Oft besteht die Absicht darin, nach einer Übersetzung für ein fehlendes Wort zu fragen. Codeswitching ist in diesem Fall ein Ausdruck von Sprachbewusstheit. Wenn sich die Frage nach Hilfe an ein anderes Kind richtet, wird dieses in den metalinguistischen Reflexionsprozess miteinbezogen.

BEISPIEL 8

(TR1204.80-87) SPRACHBETRACHTUNG RAPHAEL 6;0) UND HUGO $(5 ; 9)$

\begin{tabular}{|l|l|}
\hline Lehrperson & Na, der Hugo erklärt uns das. Was baust Du Hugo? \\
\hline Hugo & Ehm (...) Ich weiß es nicht. \\
\hline Lehrperson & Auf Deutsch nicht? \\
\hline Raphael & Ein Haus? \\
\hline Hugo & Non, de l'herbe. Comment on dit ça en allemand? \\
\hline Raphael & Gras. \\
\hline Lehrperson & Gras soll das sein. Eine Wiese? \\
\hline Raphael & Ja. \\
\hline
\end{tabular}

Die Kinder der Untersuchung haben sich an unterschiedliche sprachliche Anforderungen anpassen können. Für uns lässt sich der Wunsch ableiten, dass alle am Prozess des Frühspracherwerbs 
„Spielend“ Sprachen lernen? A. Geiger-Jaillet,
M. Vagedes Husser

BIBLIOGRAPHIE beteiligten Akteure wie Institutionen, Pädagogen und Eltern sich dahingehend orientieren, metalinguistische Fähigkeiten und flexible, funktionelle Sprachkompetenzen des Kindes anzuerkennen und aufzuwerten. Bilinguale Entwicklung findet dann statt, wenn sich in lebensnahen Kommunikations-situationen Sprachen berühren und vernetzen. Sprache ist sozial konstruiert. Bei bilingualen Lernern sind immer alle Sprachen gleichzeitig „,im Spiel“, aber es ist nicht immer „spielerisch“ einfach, ein bilingualer Lerner zu sein.

Lassen wir Gilles Forlot (2006, paragr. 36) die Ehre des Schlussworts:

Pour finir, approcher les questions linguistiques à l'école par le biais de la diversité et de la pluralité, [...] peut contribuer à la construction interculturelle des éèves, et par là même à des formes de cohésion du groupe autour de l'acceptation de la différence.

ANDRESEN H. 2002. Interaktion, Sprache und Spiel. Zur Funktion des Rollenspiels fir die Sprachentwicklung im Vorschulalter. Tübingen: Narr. DOMPMARTIN-NORMAND C. 2002. Collégiens issus de Calandreta: quelles représentations de l'occitan? In: Langage et société $2002 / 3, \mathrm{n}^{\circ} 101,35-54$.

FORLOT G. 2006. Du «fait linguistique» au «fait plurilingue» à l'école française, Éducation et sociétés plurilingues [En ligne], 38 | 2015, URL: http://esp.revues.org/554; DOI: $10.4000 /$ esp. 554

FRANQUESA F. 2015. Le système d'immersion linguistique dans les écoles de La Bressola, RECERG n ${ }^{\circ}$ - 2015 (Adresse http://recerc.casa-paisos-catalans.eu/ nicht mehr verfügbar). Unter dem Namen des Autors in den Beiträgen zum Kongress in Perpignan (2013) verfügbar

http://www.aprene.org/fr/node/2597

GEIGER-JAILLET A. (Hg.) 2010a. Lehren und Lernen in deutschsprachigen Grenzregionen. Bern: Peter Lang.

GEIGER-JAILLET A. 2010b) À propos des pratiques et des représentations: l'éducation préprimaire et le jeu en Allemagne. In: Johanne Bedard, Gilles Brougère: Feu et apprentissage: quelles relations?, Sherbrooke: Les Editions du CRP, 103-142.

LICANDRO U., LÜDTKE U. M. 2013. Peer-Interaktionen. Sprachbildung in der und durch die Gruppe. Themenheft 15 des Niedersächs. Instituts für frühkindl. Bildung und Entwicklung (nifbe), Osnabrück. Online verfügbar http://www.ifs.phil.unihannover.de/fileadmin/sonderpaedagogik/AbteilungSprachPaedagogik/Luedtke_1.pdf . 
„Spielend“ Sprachen lernen?

A. Geiger-Jaillet,

M. VAGEDES Husser

MEN (Ministère de l'éducation nationale) 2015. Programme d'enseignement de l'école maternelle. Bulletin officiel spécial $\mathrm{n}^{\circ} 2$ du 26 mars 2015. Arrêté du 18-2-2015 - J.O. du 12-3-2015 (21 Seiten) http://eduscol.education.fr/ressources-maternelle .

VAGEDES M. 2014. In zwei Sprachen spielen. 2 Bände (unveröffentlichte Masterarbeit). Universität Perpignan.

WÖRLE J. 2013. Kommunikationsstrategien und Anzeichen für Sprachbewusstheit von Kindern beim Französischlernen in einer Kindertagesstätte in der Rheinschiene. Baltmannsweiler: SchneiderVerlag Hohengehren.

\section{TRANSKRIPTIONSREGELN}

\begin{tabular}{|l|l}
\hline$(--)$ & Pause \\
\hline- & Gliederung des Redeflusses, Gedankenpause, abgebrochene Äußerung \\
\hline$*$ & nicht normgerechtes Wort oder Satzverbindung \\
\hline Xx & Unverständlich \\
\hline [Lachen, Husten] & paraverbale Kontextinformationen \\
\hline (zur Lehrperson) & Erklärung zur Handlung \\
\hline Il faut ça & franz. Äußerungen der Kinder sind kursiv gesetzt \\
\hline
\end{tabular}

FUSSNOTE

(1) Binäre Weltsicht im Französischen mit den bestimmten Artikeln le/la gegenüber der dreigeteilten Weltsicht im Deutschen mit der/die/das. 